\title{
Clinical Pharmacists in Pediatric Units
}

\author{
Abdul Kader Mohiuddin* \\ Department of Pharmacy, Bangladesh
}

*Corresponding author: Abdul Kader Mohiuddin, Department of Pharmacy, World University of Bangladesh, Bangladesh.

Received Date: July 16, 2019

Published Date: July 18, 2019

\section{Opinion}

Clinical pharmacists give valuable administrations to grown-up patients, however their advantages for pediatric and neonatal emergency clinic patients are less characterized [1]. The 2016 National Survey of Children's Health estimates that $37.5 \%$ of children in the United States have at least 1 chronic health condition, and $14.2 \%$ have required at least 1 prescription medication for 12 months or longer [2]. An estimate is that children in ancient Egypt had a $30 \%$ chance of dying before puberty, while a similar estimate in the United States in the19th century was 25\% [3]. Child an.d young person mortality have fallen in Europe, but child death rates from treatable causes including asthma, pneumonia and meningitis are higher in the UK than elsewhere in Europe, highlighting a need to better manage acutely sick children [4]. The preventable infectious disease spectrum are the major cause of death and disability in South Asia, contributing to 3.7 million deaths in children, over $60 \%$ of whom are under 5 years old. Studies on the pediatric ED disease spectrum in developing countries show that majority of chief complaints and final diagnoses are infectionrelated (up to 64\%) [5]. Even though Global under-five mortality rate dropped 53\% since 1990, the WHO estimates that approximately 5.9 million children under age five died in 2015, 16 000 every day, and respiratory infections especially pneumonia contributes about 13\% [6]. Younger children under the age of 2 in the developing world, account for nearly $80 \%$ of pediatric deaths secondary to pneumonia. Approximately a third to half of these cases lead to hospitalizations [7]. Currently there are more than 3600 therapeutic products available for the treatment of children in the United States and Canada, with approximately 20 to 30 new drugs entering the market every year [8]. According to most studies, medication errors in children are more common than in adults with a greater risk of death [9]. Medication administration error was found 2.6\% in hospitalized children of UK, 8.4\% in Argentina, 21\% in USA, $68 \%$ in India, $75 \%$ in Nigeria and around 90\% in Ethiopia at different point of time [10]. Medication mistakes caught by pharmacists included over-and under-dosing, missed portions, medication history holes, sensitivities, and close misses. Interventions to address these blunders were emphatically gotten, and actualized by doctors, with a normal acknowledgment rate of over 95\% [1]. Developmental, psychosocial, and chronic disease issues for children and adolescents may require frequent counseling and treatment visits separate from preventive care visits. Additional visits also may become necessary if circumstances suggest variations from the normal [11]. Over the last 25 years, the pharmacy profession has been engaged in organized efforts both to elevate practice and educational standards for pediatric pharmacy practice and to design medication management systems that benefit children and their families and caregivers. Moreover, alignment with pediatric clinical pharmacologists will aid in the development of new practice-based research paradigms that can be applied in the clinical setting [12]. Pediatric practice differs in many ways including, but not limited to, patient and family education efforts; pediatric dosage forms and devices; intravenous medication and enteral feeding tube compatibility consultations; fluid, electrolyte, and parenteral nutrition consults; the necessity to individualize doses (e.g., weight based); common off-label use of medications; lack of robust data informing prescribing practices; and complex age-specific pharmacokinetic and pharmacodynamic differences [13]. The special issue will expand on the needs of further advancing the care of pediatric patients, including the following: (a) addressing the need for a multi-disciplinary national pediatric pharmacotherapy collaborative practice network; (b) exploring sub-specialty needs, processes, and models of care; and (c) describing examples of collaborative practice agreements (CPAs) between clinical pharmacists, clinical pharmacologists, and pediatricians for providing comprehensive medication management (CMM) for children with special health care needs and medical complexity (CSHCN-CMC) [14]. Reviews for formulary additions should include a thorough evaluation of the available literature, 
focusing on efficacy, safety, cost, and comparison to other agents. This is especially important in pediatrics, as many medications are not adequately studied in children and must often be prescribed "off-label" [15]. Pediatric pharmacotherapy poses numerous challenges that are best suited for pharmacists with specialized training. Some of these challenges include limited data on the efficacy and safety of drug therapy in children; changes in pharmacokinetic and pharmacodynamic parameters with growth and development; dose calculations based on body weight or body surface area; manipulation of commercially available dosage forms for administration to young patients; age and developmentally appropriate medication counseling for patients as well as their caregivers; and an increased risk of adverse drug events [16]. Pediatric Pharmacy Advocacy Group (PPAG) underwrites and advocates for the contribution of pediatric pharmacists in pharmacogenomic testing and in utilizing those outcomes to give protected and compelling medication use in pediatric patients all things considered [17]. Pediatric clinical pharmacists also routinely serve on pharmacy and therapeutics committees and work closely with pharmacy information technology support. In these roles they are well positioned to recommend gene/drug pairs for implementation within an institution as well as to create clinical decision support tools that are tailor-made for prescribing physicians, physician assistants, and nurse practitioners [18]. In a 2014 survey of children's hospitals, one-third of the 88 hospitals that completed the survey had some level of pharmacist-physician collaborative drug therapy management (CDTM) in place that allowed pharmacists to modify doses and monitor therapy, with almost $75 \%$ of hospitals allowing pharmacists to initiate the first dose [2]. A pharmacist with pediatric training should provide the pharmacy department and other healthcare professionals with information on new and investigational drugs, adverse effects of and contraindications to drug therapy, compatibility and stability information, drug shortages and alternatives, dosage computations, pharmacokinetics, pharmacogenomics, and drug interactions related to the pediatric population. Pharmacists shall keep the hospital's staff and healthcare providers informed about the use of medications in the pediatric population on an ongoing basis through appropriate publications, presentations, and programs [19]. Worldwide, there is growing recognition that pharmacy education needs to be developed and improved so as to better prepare graduates to provide pediatric pharmaceutical care [20]. The 2019 Recommendations for Preventive Pediatric Health Care (Periodicity Schedule) have been approved by the American Academy of Pediatrics (AAP) and represent a consensus of AAP and the Bright Futures Periodicity Schedule Workgroup. The AAP continues to emphasize the great importance of continuity of care in comprehensive health supervision and the need to avoid fragmentation of care [11]. WHO has developed the present evidence-informed recommendations with a view to promoting the best possible clinical practices that consider the risks and benefits, acceptability, feasibility, cost and other resource implications [21]. The implementation of a unit dose dispensing model based on a dose standardization program in pediatric patients improved pharmacy workflow by decreasing drug waste, missing medication request and preparation time [22]. Nearly 20\% (or 17 million) of children under the age of 18 have at least one special need [23,24]. The Post-Acute Acuity Rating for Children, intended to reflect medical severity based on age, reason for admission, diagnoses, dependence in activities of daily living, and technology reliance for children admitted to post-acute care rehabilitation hospitals, has been found to be a comparable measurement of medical complexity for pediatric outpatient care [25]. Potential network infrastructures for collaborative practice focused on CSHCN populations, common language and terminology for $\mathrm{CMM}$, and clinical pharmacist workforce estimates are provided. Embedding clinical pharmacists into the Patient-Centered Medical Home (PCMH) to provide comprehensive medication management (CMM) through collaborative practice agreements (CPAs) for children, especially for CSHCN, can improve outcomes, enhance the experience of care for families, and reduce the cost of care [26]. Literature suggests that up to $60 \%$ of all medication errors occur during transitions of care and nearly 1 in every 5 hospital discharges may result in readmission. A prospective study to investigate medication discrepancies for the discharge of pediatric patients reported that $8 \%$ of medications contained discrepancies between documentation sources [27]. Top opportunities identified to improve medication safety include using kilogram-only weight-based dosing, optimizing computerized physician order entry by using clinical decision support, developing a standard formulary for pediatric patients while limiting variability of medication concentrations, using pharmacist support within EDs, enhancing training of medical professionals, systematizing the dispensing and administration of medications within the ED, and addressing challenges for home medication administration before discharge [28]. Antibiotics are the class of medications with the most frequent interventions (34\%). About half of the interventions in this class are pharmacokinetic evaluations to optimize drug levels and minimize toxicities. Agent recommendations accounted for $15 \%$ of antibiotic interventions, which included de-escalation of therapy as part of antimicrobial stewardship in PICU [29]. Dose optimization and IV to PO conversion play a large role in stewardship efforts. To ensure that patients are on the proper dose to optimally treat an infection, the dosage of some antibiotics may need to be increased. The renal dosing protocol allows pharmacists to automatically adjust medications, including certain antibiotics, to avoid adverse effects in patients with fluctuations in renal function. The clinical decision support system can enable pharmacists to compare and leverage patient data in near real-time from the different systems within the hospital, for example, pharmacy and microbiology [30]. A unique partnership between nurses and pharmacists facilitated the discharge process for clinically complex children [31]. There is a need to expand clinical pharmacy services to cover the ambulatory pediatric cancer patients. Developing pediatric hematologyoncology clinical pharmacy services to cover the outpatient setting is essential to ensure continuity of care and to optimize therapeutics [32]. A pharmacist-led, educational program significantly improves knowledge of chemotherapy administration and monitoring in 
pediatric oncology nurses and was well received by participants as an additional training opportunity [33].

\section{Acknowledgement}

I'm thankful to Dr. Arinze Nkemdirim Okere, Associate Professor of Pharmacy, Clinical and Administrative Sciences, Florida A\&M University for his precious time to review my letter to the editor and for his thoughtful suggestions. I'm also grateful to seminar library of Faculty of Pharmacy, University of Dhaka and BANSDOC Library, Bangladesh for providing me books, journal and newsletters.

\section{Conflict of Interest}

The author declares that he has no competing interests.

\section{References}

1. Mohiuddin AK (2019) Pharmacists in Public Health: Scope in Home and Abroad. SOJ Pharm Sci 6(1):1-23.

2. Tran V, McMunn A, Sterner-Allison J, Stoffella S (2019) Credentialing of Pediatric Pharmacists in Collaborative Drug Therapy Management. J Pediatr Pharmacol Ther 24(2): 172-174.

3. Rieder M (2010) If children ruled the pharmaceutical industry: the need for pediatric formulations. Drug News Perspect 23(7): 458-464.

4. Jones CH, Neill S, Lakhanpaul M, Roland D, Singlehurst-Mooney H, et al. (2014) Information needs of parents for acute childhood illness: determining 'what, how, where and when' of safety netting using a qualitative exploration with parents and clinicians. BMJ Open 4(1): e003874.

5. Atiq H, Siddiqui E, Bano S, Feroze A, Kazi G, et al. (2015) The pediatric disease spectrum in emergency departments across Pakistan: data from a pilot surveillance system. BMC Emerg Med 15 Suppl 2: S11.

6. Tabbal AOA, Humedi SSA (2017) Surveillance of the Most Prevalent Medical Diseases among Pediatric Age Groups and Evaluation of the Control Measures Used at Tabuk Hospitals, Saudi Arabia. Open Access Maced J Med Sci 5(2): 182-187.

7. Ebeledike C, Ahmad T (2019) Pediatric Pneumonia. In: StatPearls [Internet]. Treasure Island (FL): StatPearls Publishing.

8. Rieder M. Adverse Drug Reactions in Children: Pediatric Pharmacy and Drug Safety. J Pediatr Pharmacol Ther 24(1): 4-9.

9. Izadpanah F, Nikfar S, Bakhshi Imcheh F, Amini M, Zargaran M (2018) Assessment of Frequency and Causes of Medication Errors in Pediatrics and Emergency Wards of Teaching Hospitals Affiliated to Tehran University of Medical Sciences (24 Hospitals). J Med Life 11(4): 299-305.

10. Baraki Z, Abay M, Tsegay L, Gerensea H, Kebede A, et al. (2018) Medication administration error and contributing factors among pediatric inpatient in public hospitals of Tigray, northern Ethiopia BMC Pediatr 18(1): 321.

11. Committee on Practice and Ambulatory Medicine; Bright Futures Periodicity Schedule Workgroup. 2019 Recommendations for Preventive Pediatric Health Care. Pediatrics 143(3): e20183971.

12. Parrish RH 2nd, Van den Anker J, Benavides S (2019) Where are the Ambulatory Care Pediatric Pharmacists? Children (Basel). 6(2): E25.

13. Blackmer AB, Siu A, Thornton A, Johnson PN, Nichols KR, et al. (2019) Academic Careers in Pediatric Pharmacy: Part 2-Academic Advancement. J Pediatr Pharmacol Ther 24(3): 183-193.

14. Bhatt-Mehta V, Buck ML, Chung AM, Farrington EA, Hagemann TM, et al. (2012) Recommendations for Meeting the Pediatric Patient's Need for a Clinical Pharmacist: A Joint Opinion of the Pediatrics Practice and Research Network of the American College of Clinical Pharmacy and the Pediatric Pharmacy Advocacy Group. J Pediatr Pharmacol Ther 17(3): 281-291.

15. Austin JP, Gunden S, Hoffner W, Ismail L, Mendez S, et al. (2019) The Value of Pediatricians on Pharmacy and Therapeutics Committees. PT 44(1): $2-4$
16. Thompson K, Gammal RS, Benefield EC, Condren ME (2019) Ambulatory Care Training Within Postgraduate Year 2 Pediatric Pharmacy Residency Programs. J Pediatr Pharmacol Ther 24(3): 214-219.

17. Kennedy MJ, Phan H, Benavides S, Potts A, Sorensen S (2011) The role of the pediatric pharmacist in personalized medicine and clinical pharmacogenomics for children: pediatric pharmacogenomics working group. J Pediatr Pharmacol Ther 16(2): 118-222.

18. Brown JT, Gregornik D, Kennedy MJ, Advocacy and Research Committees (2018) The Role of the Pediatric Pharmacist in Precision Medicine and Clinical Pharmacogenomics for Children. J Pediatr Pharmacol Ther 23(6): 499-501.

19. Eiland LS, Benner K, Gumpper KF, Heigham MK, Meyers R, et al. (2018) ASHP-PPAG Guidelines for Providing Pediatric Pharmacy Services in Hospitals and Health Systems. J Pediatr Pharmacol Ther 23(3): 177-191.

20. Mukattash TL, Jarab AS, Abu-Farha RK, Alefishat E, McElnay JC (2018) Pharmaceutical Care in Children: Self-reported knowledge, attitudes and competency of final-year pharmacy students in Jordan. Sultan Qaboos Univ Med J 18(4): e468-e475

21. (2012) Recommendations for Management of Common Childhood Conditions: Evidence for Technical Update of Pocket Book Recommendations: Newborn Conditions, Dysentery, Pneumonia, Oxygen Use and Delivery, Common Causes of Fever, Severe Acute Malnutrition and Supportive Care. Geneva: World Health Organization.

22. Topolski J, Tanweer A, Rabin M (2018) Improving pharmacy workflow through the implementation of a unit dose dispensing model based on a dose standardization program in pediatric patients. J Pediatr Pharmacol Ther 23(3): 227-270.

23. (2019) Health Resources and Services Administration. Maternal and Child Health Bureau Children with Special Health Care Needs.

24. Bethell CD, Read D, Blumberg SJ, Newacheck PW (2008) What is the prevalence of children with special health care needs? Toward an understanding of variations in findings and methods across three national surveys. Matern Child Health J 12(1): 1-14.

25. 0 Brien JE, Dumas HM (2015) Development and Initial Psychometric Evaluation of the Post-Acute Acuity Rating for Children. Rehabil Res Pract 2015: 841523.

26. Parrish Ii RH, Casher D, Van den Anker J, Benavides S (2019) Creating a Pharmacotherapy Collaborative Practice Network to Manage Medications for Children and Youth: A Population Health Perspective. Children (Basel) 6(4): E58.

27. Gattari TB, Krieger LN, Hu HM, Mychaliska KP (2015) Medication Discrepancies at Pediatric Hospital Discharge. Hosp Pediatr 5(8): 439445.

28. Benjamin L, Frush K, Shaw K, Shook JE, Snow SK; American Academy of Pediatrics Committee on Pediatric Emergency Medicine; American College of Emergency Physicians Pediatric Emergency Medicine Committee; Emergency Nurses Association Pediatric Emergency Medicine Committee (2018) Pediatric Medication Safety in the Emergency Department. Pediatrics 141(3): e20174066.

29. Larochelle JM, Ghaly M, Creel AM (2012) Clinical pharmacy faculty interventions in a pediatric intensive care unit: an eight-month review. J Pediatr Pharmacol Ther 17(3): 263-269.

30. Nguyen V, Sarik DA, Dejos MC, Hilmas E (2018) Development of an Interprofessional Pharmacist-Nurse Navigation Pediatric Discharge Program. J Pediatr Pharmacol Ther 23(4): 320-328.

31. Calloway S, Akilo HA, Bierman K (2013) Impact of a clinical decision support system on pharmacy clinical interventions, documentation efforts, and costs. Hosp Pharm 48(9): 744-752.

32. Tuffaha HW, Abdelhadi O, Omar SA (2012) Clinical pharmacy services in the outpatient pediatric oncology clinics at a comprehensive cancer center. Int J Clin Pharm 34(1): 27-31.

33. Fisher CM, Kim AJ, Elder JJ (2017) Impact of a Pharmacist-Led Chemotherapy Education Program on the Knowledge of Pediatric Hematology/Oncology Nurses. J Pediatr Pharmacol Ther 22(5): 332337. 